\title{
Cohort study of consistency between the compliance with guidelines for chemotherapy-induced nausea and vomiting and patient outcome
}

Masahiro Inoue ${ }^{1,2}$, Manabu Shoji ${ }^{2,3}$, Naomi Shindo $^{2}$, Kazunori Otsuka ${ }^{1,2}$, Masatomo Miura ${ }^{3}$ and Hiroyuki Shibata ${ }^{12^{*}}$

\begin{abstract}
Background: Chemotherapy-induced nausea and vomiting is one of the most influential factors that affect patient quality of life; thus, preventing this adverse event could lead to better patient outcome. Standard preventive guidelines for antiemetic treatment have already been established based on the emetogenicity of chemotherapeutic agents. It is important that compliance with in-house guidelines and their effect on patient outcome is monitored.

Methods: In 3 years since the Akita university hospital antiemetic guidelines were outlined, we assessed the incidence of chemotherapy-induced nausea and vomiting using the antiemesis tool of the Multinational Association of Supportive Care in Cancer. Compliance of the guidelines was extracted from the hospital clinical record, and the chemotherapyinduced nausea and vomiting was examined by the patient reported outcome.

Results: Seventy-three patients answered the questionnaire. The overall compliance rate with the guidelines for early nausea and vomiting was $98.6 \%$ and with the delayed nausea and vomiting was $87.7 \%$. The complete response rate for the early and delayed chemotherapy-induced nausea and vomiting was $77.8 \%$ and $73.8 \%$, respectively. The overall relative risk of early nausea and vomiting was $0.22(P<0.05)$, whereas the relative risk for delayed nausea and vomiting was $2.09(P<0.05)$. Breakthrough vomiting was observed in 3 cases in the low-risk group only. These data suggest that delayed nausea and vomiting is difficult to prevent, particularly in the low-risk group. Further, it seems that the individual sensitivity for emetogenicity might differ among patients.
\end{abstract}

Conclusions: In addition to standard prevention guidelines based on emetogenicity, individual care based on patient reports should be considered for the complete prevention of chemotherapy-induced nausea and vomiting.

Keywords: Chemotherapy-induced nausea and vomiting, Guidelines, Emetogenicity

\section{Background}

One of the most devastating effects on the quality of life (QOL) of cancer patients is chemotherapy-induced nausea and vomiting (CINV). CINV is believed to affect $70 \%-80 \%$ of patients that receive cancer chemotherapy [1]. In addition, even effective chemotherapy may be stopped because of severe CINV, and the clinical losses may be significant in these cases [2].

\footnotetext{
* Correspondence: hiroyuki@med.akita-u.ac.jp

'Department of Clinical Oncology, Graduate School of Medicine, Akita University, Akita, Japan

2Division of Chemotherapy for Outpatient, Akita University, Akita, Japan Full list of author information is available at the end of the article
}

The underlying mechanisms that cause CINV have been determined gradually, and many neurotransmitters such as 5-hydroxytryptamine (5- $\mathrm{HT}_{3}$, serotonin) and substance $P$ are involved in CINV [3]. 5-HT3 receptor antagonists (5-HT3RA) such as granisetron, ondansetron, and palonosetron have been approved for the treatment of CINV [4]. In addition, aprepitant was approved as a substance P blocker and a neurokinin 1 (NK1) receptor antagonist (NK1RA) for the prevention of acute and delayed CINV [4]. These agents have greatly improved patient outcome regarding CINV. For example, it was reported that a complete response of CINV occurred in $53.6 \%-53.7 \%$ of patients that received moderately 
emetogenic chemotherapy using palonosetron plus dexamethasone (DEX) [5]. It was also reported that, among patients receiving cisplatin-based chemotherapy, the advantage achieved by the use of aprepitant was 20 percentage points [6].

Since the Multinational Association of Supportive Care in Cancer (MASCC) released their antiemetic guidelines in 1998, the American Society of Clinical Oncology (ASCO) and the National Comprehensive Cancer Network (NCCN) also released guidelines for the prevention and treatment of CINV [7]. Antiemetic strategies primarily using 5-HT3RA, NK1RA, and DEX have been established and recommended based on four emetogenicities (high, moderate, low, and minimal risk) for each chemotherapeutic agent [7]. In individual institutions, in-house guidelines for the prevention and treatment of CINV according to the MASCC, ASCO, and NCCN guidelines have been created and implemented, including in our hospital. Added to these guidelines, it is very important to monitor both patient outcome and their compliance with the guidelines [8]. However, because awareness of the occurrence of CINV depends only on patient declarations, it is difficult to know the severity of the CINV without patient-reported outcomes. In the current study, we assessed the consistency between the CINV compliance guidelines set in 2010 in Akita University Hospital and the outcome of patients visiting the Division of Chemotherapy for Outpatients. In this observational study, we revealed that the individual monitoring of CINV, even in low-risk emetogenicity patients, is very important to improve patient QOL during chemotherapy.

\section{Methods}

Patients who visited the Division of Chemotherapy for Outpatients, Akita University Hospital, between November 2013 and March 2014 were asked about their early and delayed CINV using the Japanese version of the MASCC Antiemesis Tool (MAT) [9], which was administered at a usual medical examination using a questionnaire. The grade of nausea was rated from 1 to 10 (1, minimum; 10 , maximum), and the number of times of vomiting was recorded by the patients themselves. Questions were asked at any time during treatment, but only once for each patient. Conducted chemotherapeutic and anti-emetic agents were extracted from electronic clinical record of hospital, and the compliance of guideline was examined. Further, comparison was made between the patient reported outcome and the compliance of guideline (Figure 1). Stat Mate III (ATMS, Tokyo, Japan) was used to calculate the relative risk. The level of statistical significance was set as $P<0.05$. This retrospective study was approved by the Ethics Committee of the School of Medicine of Akita University. Written informed consent for participation in the study was obtained from participants.

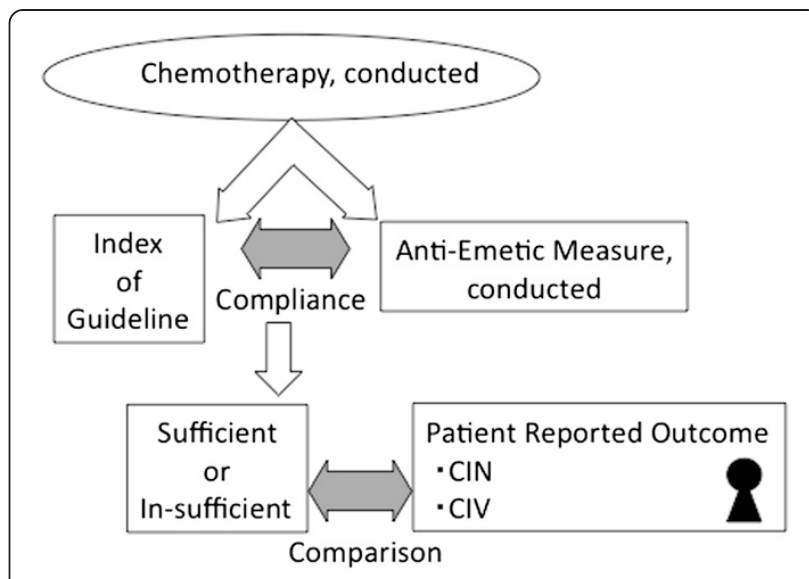

Figure 1 Schematic presentation of this study. Comparison between compliance of anti-emetic guideline and patient reported outcome.

\section{Results and discussion}

Patients

The patients included 36 males and 37 females aged 2879 years, with a median age of 62 years (Table 1). The patients were diagnosed with the malignancies listed in Table 1. Colorectal cancer was most frequent (16/73), followed by breast (13/73), lung (9/73), and gastric cancers (9/73). All participants were diagnosed as suitable for chemotherapy as outpatients, without any clinical signs of brain metastasis or intestinal obstructions. The performance status of all participants was below 2, according to the Eastern Cooperative Oncology Group. The detailed clinical information such as clinical stages,

Table 1 Patients' characteristics

\begin{tabular}{|c|c|}
\hline Number of participant & 73 \\
\hline Age (median) & $28-79(62)$ \\
\hline Male: Female & 36: 37 \\
\hline Chemotherapy naïve patients & 3 \\
\hline \multicolumn{2}{|l|}{ Primary leasion } \\
\hline Esophageal cancer & 2 \\
\hline Gastric cancer & 9 \\
\hline Colorectal cancer & 16 \\
\hline Bile duct carcinoma & 1 \\
\hline Pancreatic cancer & 7 \\
\hline Breast cancer & 13 \\
\hline Lung cancer & 9 \\
\hline Malignant lymphoma & 3 \\
\hline Acute lymphoblastic leukemia & 1 \\
\hline Malignant myeloma & 2 \\
\hline Prostate cancer & 5 \\
\hline Renal cell carcinoma & 1 \\
\hline Cancer of unknown primary & 4 \\
\hline
\end{tabular}


previous treatment is listed in Additional file 1: Table S1. Chemotherapy naïve, and the other patients experienced $\geq 2$ cycles of chemotherapy.

\section{Regimens}

The regimens used to treat the patients and their emetic risks are listed in Table 2. The most frequent regimen was docetaxel alone (9/73), followed by gemcitabine (8/73). The regimens were divided into four emetic risk groups: high, moderate, low, and minimal risks. The number of patients receiving high-, moderate-, low-, and minimalrisk regimens was $11,17,38$, and 7 , respectively.

\section{Compliance with the antiemetic guideline}

The compliance of the antiemetic guideline was examined. Our anti-emetic guideline is indicated in Table 3. Sufficient antiemetic treatments were performed for the minimal-risk regimens in all patients. In the low-risk group, all except 1 case received sufficient treatments; one under treatment was performed in the early phase, and no under treatments were performed in the delayed phase. For the moderate-risk group, sufficient treatments were performed in 14 of cases (82.4\%); no under treatments occurred in the early phase, whereas three under treatments were performed in the delayed phase. For the high-risk group, sufficient treatments were performed in 6 cases (54.5\%); no under treatments occurred in the early phase, whereas five under treatments were performed in the delayed phase. The overall compliance rate was $87.7 \%$; the compliance rate was $98.6 \%$ with early phase CINV. The compliance rate for delayed phase CINV was $87.7 \%$; specifically, $97.4 \%$ in the low-risk group, $82.4 \%$ in the moderate group, and $54.5 \%$ in the high-risk group.

\section{Comparison between patient outcome and compliance with the guidelines}

We next compared the patient-reported CINV and the compliance of guideline. As shown in Figure 2, early CINV was not prevented in $22.2 \%$ of cases, although the sufficient antiemetic treatment against was conducted. However, early CINV was not prevented at all in the under treatment case. The overall relative risk for compliance with the guidelines was calculated to be $0.22(P<$ 0.05). Specifically, in the minimal-risk group, CIN was not prevented in one of 7 cases (14.3\%), in spite of sufficient treatment (the grade of nausea was 2 (G2)). In the lowrisk group, CIN was not prevented in 8 of 37 cases $(21.6 \%)$ (G1 to G5; median = G2.5). Among these, one patient vomited once (2.7\%). In the moderate-risk group, CIN was not prevented in 2 of 7 cases (11.8\%) (G2 to G4; median = G3), but no CIV was observed. In the high-risk group, CIN was not prevented in 5 of 11 cases $(45.5 \%)$ (G1 to G6; median = G3), but no CIV was observed.
Table 2 Treatment regimens and emetogenisity

\begin{tabular}{|c|c|c|c|}
\hline Emetogenisity & $\begin{array}{l}\text { Index of anti-emetic } \\
\text { guideline }\end{array}$ & Regimen & Number \\
\hline \multirow{5}{*}{$\begin{array}{l}\text { Minimal } \\
(\mathrm{n}=7)\end{array}$} & \multirow[t]{5}{*}{ Early; (-), Delayed; (-) } & Tmab + VNR & 2 \\
\hline & & Tmab & 2 \\
\hline & & VNR & 1 \\
\hline & & $M T X+V C R+P S L$ & 1 \\
\hline & & temsirolimus & 1 \\
\hline \multirow{14}{*}{$\begin{array}{l}\text { Low } \\
(n=38)\end{array}$} & \multirow[t]{14}{*}{ Early; DEX, Delayed; (-) } & GEM & 8 \\
\hline & & S-1 + GEM & 1 \\
\hline & & nab-PTX + GEM & 1 \\
\hline & & nab-PTX & 3 \\
\hline & & Tmab + PTX & 2 \\
\hline & & PTX & 1 \\
\hline & & DTX + S-1 & 2 \\
\hline & & DTX + EMP & 1 \\
\hline & & DTX + PSL & 4 \\
\hline & & DTX & 9 \\
\hline & & eribulin & 1 \\
\hline & & Bmab + sLV5FU2 & 1 \\
\hline & & sLV5FU2 & 3 \\
\hline & & VTD & 1 \\
\hline \multirow{10}{*}{$\begin{array}{l}\text { Moderate } \\
(n=17)\end{array}$} & \multirow{10}{*}{$\begin{array}{l}\text { Early; NK1RA + 5-HT } 3 \text { RA + } \\
\text { DEX, or 5-HT } 3 \text { RA + DEX, } \\
\text { Delayed; NK1RA + } \\
5-\mathrm{HT}_{3} \mathrm{RA}+\mathrm{DEX} \text {, or } \\
5-\mathrm{HT}_{3} \mathrm{RA}+\mathrm{DEX}\end{array}$} & Bmab + CBDCA + PTX & 3 \\
\hline & & Pmab + FOLFOX6 & 2 \\
\hline & & Bmab + CapeOX & 1 \\
\hline & & CapeOX & 3 \\
\hline & & SOX & 1 \\
\hline & & Bmab + FOLFIRI & 1 \\
\hline & & $B m a b+I R I S$ & 1 \\
\hline & & IRIS & 2 \\
\hline & & CPT-11 & 2 \\
\hline & & VCD & 1 \\
\hline \multirow{4}{*}{$\begin{array}{l}\text { High } \\
(n=11)\end{array}$} & \multirow{4}{*}{$\begin{array}{l}\text { Early; NK1RA + 5-HT, } \mathrm{RA}+ \\
\text { DEX, Delayed; NK1RA + } \\
\text { 5-HT }{ }_{3} \mathrm{RA}+\mathrm{DEX}\end{array}$} & CDDP + CPT-11 & 4 \\
\hline & & FEC & 2 \\
\hline & & EC & 2 \\
\hline & & $\mathrm{R}-\mathrm{CHOP}$ & 3 \\
\hline
\end{tabular}

Tmab; Trastuzumab, VNR; vinorelbine, MTX; Methotrexate, VCR; Vincristine, PSL; Prednisolone, GEM; Gemcitabine, nab-PTX; nab-Paclitaxel, DTX; Docetaxel, EMP; estramustine, Bmab; Bevacizumab, sLV5FU2; simplified biweekly 5-FU \& leucovorin, VTD; bortezomib, thalidomide, dexamethasone, CBDCA; Carboplatin, FOLFOX6; combination of Oxaliplatin and 5FU, CapeOX; capecitabine plus intermittent oxaliplatin, SOX; S-1 plus intermittent oxaliplatin, FOLFIRI; combination of Irinotecan and 5FU IRIS; CPT-11; Irinotecan, VCD; bortezomib, Cyclophosphamide, dexamethasone, CDDP; Cisplatin, FEC; 5FU, Epirubicin, Cyclophosphamide, EC; Epirubicin, Cyclophosphamide, R-CHOP; Rituximab, Cyclophosphamide, Doxorubicin, Vincristine, Prednisolone.

Delayed CINV was not prevented in 17 of the 65 cases (26.2\%), even though sufficient antiemetic treatments were conducted (Figure 3). In the under treatment cases, CINV (G1) was not prevented in 1 of 8 cases (12.5\%). 
Table 3 Compliance of antiemetic treatment

\begin{tabular}{|c|c|c|c|c|}
\hline Emetogenisity & Actual measure (Early) & Actual measure (Delayed) & $\mathrm{n}$ & Compliance \\
\hline \multirow[t]{4}{*}{ Minimal } & $5-\mathrm{HT}_{3} \mathrm{RA}+\mathrm{DEX}$ & no & 2 & Sufficient \\
\hline & $5-\mathrm{HT}_{3} \mathrm{RA}$ & no & 1 & Sufficient \\
\hline & DEX & no & 1 & Sufficient \\
\hline & no & no & 3 & Sufficient \\
\hline \multirow[t]{6}{*}{ Low } & $5-\mathrm{HT}_{3} \mathrm{RA}+\mathrm{DEX}$ & no & 29 & Sufficient \\
\hline & $5-\mathrm{HT}_{3} \mathrm{RA}+\mathrm{DEX}$ & DEX & 3 & Sufficient \\
\hline & $5-\mathrm{HT}_{3} \mathrm{RA}+\mathrm{DEX}$ & D2RA & 1 & Sufficient \\
\hline & DEX & DEX & 1 & Sufficient \\
\hline & DEX & no & 3 & Sufficient \\
\hline & no & no & 1 & Insufficient \\
\hline \multirow[t]{7}{*}{ Moderate } & $5-\mathrm{HT}_{3} \mathrm{RA}+\mathrm{DEX}$ & DEX & 6 & Sufficient \\
\hline & $5-\mathrm{HT}_{3} \mathrm{RA}+\mathrm{DEX}$ & NK1RA + DEX & 3 & Sufficient \\
\hline & $\mathrm{NK} 1 \mathrm{RA}+5-\mathrm{HT}_{3} \mathrm{RA}+\mathrm{DEX}$ & NK1RA & 2 & Sufficient \\
\hline & $\mathrm{NK} 1 \mathrm{RA}+5-\mathrm{HT}_{3} \mathrm{RA}+$ steroid $(\mathrm{MM})$ & NK1RA & 1 & Sufficient \\
\hline & $\mathrm{NK} 1 \mathrm{RA}+5-\mathrm{HT}_{3} \mathrm{RA}+\mathrm{DEX}$ & $5-\mathrm{HT}_{3} \mathrm{RA}+\mathrm{DEX}+\mathrm{NK} 1 \mathrm{RA}$ & 1 & Sufficient \\
\hline & $5-\mathrm{HT}_{3} \mathrm{RA}+\mathrm{DEX}$ & no & 3 & Insufficient \\
\hline & $5-\mathrm{HT}_{3} \mathrm{RA}+\mathrm{DEX}$ & $\mathrm{DEX}+\mathrm{D} 2 \mathrm{RA}$ & 1 & Sufficient \\
\hline \multirow[t]{5}{*}{ High } & $\mathrm{NK} 1 \mathrm{RA}+5-\mathrm{HT}_{3} \mathrm{RA}+\mathrm{DEX}$ & NK1RA & 2 & Insufficient \\
\hline & $\mathrm{NK} 1 \mathrm{RA}+5-\mathrm{HT}_{3} \mathrm{RA}+$ steroid $(\mathrm{ML})$ & NK1RA & 2 & Sufficient \\
\hline & $\mathrm{NK} 1 \mathrm{RA}+5-\mathrm{HT}_{3} \mathrm{RA}+$ steroid $(\mathrm{ML})$ & $\mathrm{NK} 1 \mathrm{RA}+\mathrm{D} 2 \mathrm{RA}$ & 1 & Sufficient \\
\hline & $5-\mathrm{HT}_{3} \mathrm{RA}+\mathrm{DEX}$ & DEX & 2 & Insufficient \\
\hline & $5-\mathrm{HT}_{3} \mathrm{RA}+\mathrm{DEX}$ & no & 1 & Insufficient \\
\hline
\end{tabular}

5- $\mathrm{HT}_{3} \mathrm{RA} ; 5-\mathrm{HT}_{3}$ receptor antagonist, DEX; Dexamethasone, D2RA; Dopamine receptor D2 receptor antagonist, NK1RA; Neurokinin 1 receptor antagonist, $\mathrm{MM}$; multiple myeloma, $\mathrm{ML}$; malignant lymphoma.

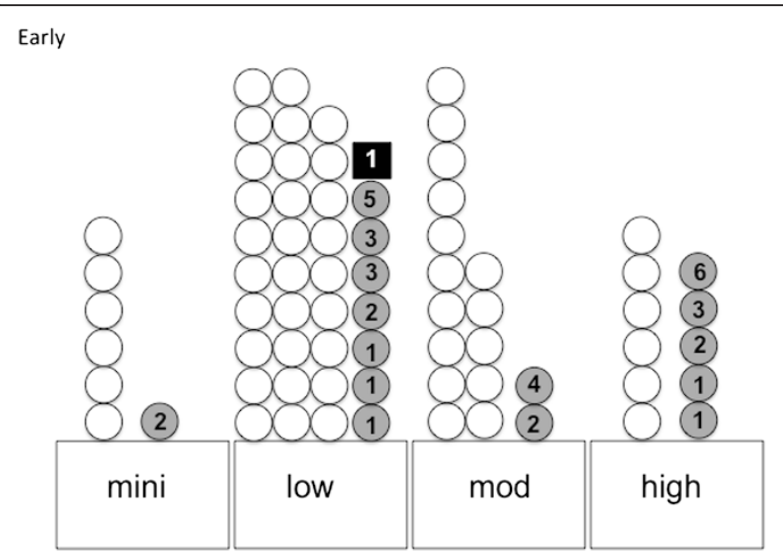

Figure 2 Patient-reported outcome regarding early CINV in patients treated with sufficient antiemetic guidelines. Mini, minimal-risk group; mod, moderate-risk group. An open circle indicates the case with a complete response. The shaded circle indicates CIN (the number corresponds to the grade of nausea). The closed rectangle indicates CIV (the number corresponds to the number of times of vomiting).

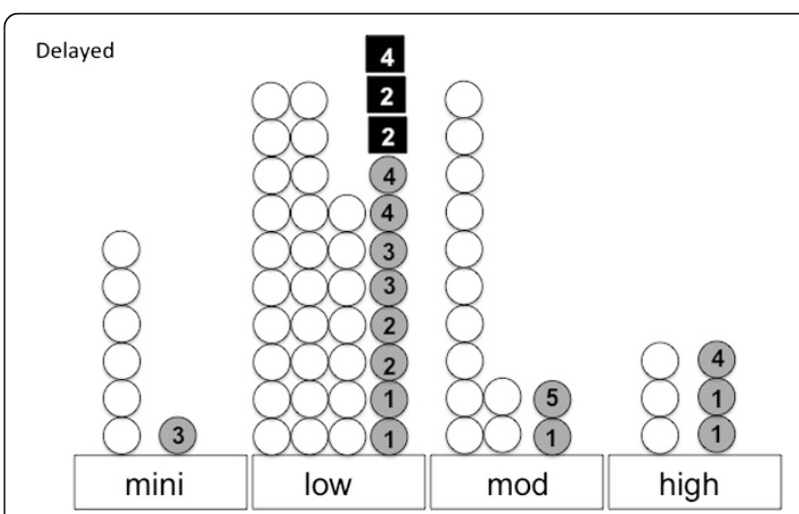

Figure 3 Patient-reported outcome regarding delayed CINV in patients treated with sufficient antiemetic guidelines. Mini, minimal-risk group; mod, moderate-risk group. An open circle indicates the case with a complete response. The shaded circle indicates CIN (the number corresponds to the grade of nausea). The closed rectangle indicates CIV (the number corresponds to the number of times of vomiting). 
The overall relative risk for delayed CINV was $2.22(P<$ 0.05). Specifically, in the minimal-risk group, CIN was not prevented in 1 of 7 cases (14.3\%) (G3). In the lowrisk group, CIN was not prevented in 11 of 38 cases $(29.0 \%)($ G1 to G4; median = G3). Among these, three patients vomited (two patients twice and one patient once). In the moderate-risk group, CIN was not prevented in 2 of 14 cases (14.3\%) (G1 to G5; median = G3), but there were no instances of vomiting. In the high-risk group, CIN was not prevented in 3 of 6 cases $(50 \%)($ G1 to G4; median = G4), but there was no vomiting.

In this observational study, CIV occurred only in the patients that received low emetogenic chemotherapeutic agents. One male patient (71 years old) that reported early CIV was treated for the second time with nabpactlitaxel for his carcinoma of unknown origin, mediastinal lymph node metastasis, and pleural dissemination. The remaining three patients claimed breakthrough CIV. Of these, one female patient (42 years old) was treated for the fourth time with eribulin for breast cancer with bone and liver metastases. Another female patient (61 years old) was treated with docetaxel for the second time for lung cancer with asymptomatic and multiple micrometastases in the brain. Finally, one male patient (55 years old) was treated for the twelfth time with docetaxel for lung cancer and cancerous pleuritis. The compliance rate of our hospital was better than that reported previously [10]. Preventive treatment against early CINV was almost completely effective; however, the prevention of delayed CINV was not sufficient in the high-risk group according to the current guidelines. It is necessary to prevent delayed CINV. According to the patient-reported outcome, delayed CINV occurred in only $12.5 \%$ of the patients that were treated with antiemetic agents insufficiently. However, early and delayed CIN occurred in $22.2 \%$ and $26.2 \%$ of patients, respectively, in spite of conduction of sufficient anti-emetic treatment, particularly, in the high-risk group, early and delayed CIN occurred in $45.5 \%$ and $50.0 \%$ of patients, respectively. However, the grade of CIN was not so severe (The median grade of early $\mathrm{CIN}=\mathrm{G} 3$. and delayed CIN = G4). Moreover, no CIV (breakthrough CIV) was reported. The guidelines for the prevention of CINV in the high-risk group appeared to be effective to comparable extent. In the low-risk group, early and delayed CINV occurred in $21.6 \%$ and $28.9 \%$ of patients, respectively, in spite of sufficient anti-emetic treatment (The median grade of the early and the delayed CIN = G2.5 and G3, respectively). Moreover, 3 cases of breakthrough $\mathrm{CIV}$ were reported. Among these, all three vomited several times. These data suggest that compliance with the guideline alone could not prevent emetogenicity. Consistent with this, a previous study also reported that sufficient measures could not prevent breakthrough CIV completely, even in low-risk patients [11]. In addition, some reports have discussed the difficulty of prevention using antiemetic guidelines for delayed CINV $[12,13]$. In particular, prevention in low-risk patients remains controversial.

Current guidelines are based primarily on the emetogenicity of chemotherapeutic agents. Data suggested that individual differences in the sensitivity to antiemetic treatments may occur. There are also too many individual parameters, including exposure to chemotherapy, alcohol use, age, and gender [14,15]. As such, data should be gathered and analyzed regarding CINV cases. Such factors may include individual sensitivities to the preventive agents, tumor status, and the physical condition of patient. Refining the antiemetic measures is also necessary; accordingly, some methods have been proposed $[13,16]$. However, the establishment of the personalized precautions as well as the standard one is likely to be necessary for the complete prevention of CINV.

\section{Conclusions}

The generation of antiemetic guidelines might contribute toward patient compliance with antiemetic measures. However, complete prevention remains challenging because individual factors should be considered.

\section{Additional file}

Additional file 1: Table S1. Detailed characteristics of participants.

\section{Abbreviations}

QOL: Quality of life; CINV: Chemotherapy-induced nausea and vomiting; 5-HT3: 5-hydroxytryptamine; 5-HT3RA: 5-HT3 receptor antagonists; NK1: Neurokinin 1; NK1RA: NK1 receptor antagonist; MASCC: Multinational association of supportive care in cancer; ASCO: the American society of clinical oncology; NCCN: National comprehensive cancer network; DEX: Dexamethasone; MAT: MASCC antiemesis tool.

\section{Competing interests}

The authors declare that they have no competing interests.

\section{Authors' contributions}

MI, MS, and NS gathered the patient data. MS, NS, KO, MM, and HS set the in-house guidelines. HS planned the study and wrote the manuscript. All authors read and approved the final manuscript.

\section{Authors' information}

$\mathrm{MI}$ is an assistant professor, $\mathrm{KO}$ is a lecturer, and $\mathrm{HS}$ is a professor in the Department of Clinical Oncology. MM is a professor in the Department of Pharmacy. HS is a Japanese Society of Medical Oncology certified specialist in Medical Oncology. MS was Board-Certified as a Pharmacist in Oncology Pharmacy by the Japanese Society of Hospital Pharmacist. NS is a Japanese Nursing Association-certified nurse for cancer chemotherapy.

\section{Acknowledgments}

The authors would like to thank Enago (http://www.enago.jp) for English language review. 


\section{Author details}

'Department of Clinical Oncology, Graduate School of Medicine, Akita University, Akita, Japan. ${ }^{2}$ Division of Chemotherapy for Outpatient, Akita University, Akita, Japan. ${ }^{3}$ Department of Pharmacy, Akita University, Akita, Japan.

Received: 9 October 2014 Accepted: 12 March 2015

Published online: 28 March 2015

\section{References}

1. Morran C, Smith DC, Anderson DA, McArdle CS. Incidence of nausea and vomiting with cytotoxic chemotherapy: a prospective randomised trial of antiemetics. Br Med J. 1979;1:1323-4

2. Richardson $J$, Marks G, Levine A. The influence of symptoms of disease and side effects of treatment on compliance with cancer therapy. J Clin Oncol. 1988;6:1746-52.

3. Baker PD, Morzorati SL, Ellett ML. The pathophysiology of chemotherapyinduced nausea and vomiting. Gastroenterol Nurs. 2005;28:469-80.

4. Basch E, Prestrud AA, Hesketh PJ, Kris MG, Feyer PC, Somerfield MR, et al. American Society of Clinical Oncology guideline for antiemetics in oncology: update 2006. J Clin Oncol. 2006;24:2932-47.

5. Aapro M, Fabi A, Nolè F, Medici M, Steger G, Bachmann C, et al. Doubleblind, randomised, controlled study of the efficacy and tolerability of palonosetron plus dexamethasone for 1 day with or without dexamethasone on days 2 and 3 in the prevention of nausea and vomiting induced by moderately emetogenic chemotherapy. Ann Oncol. 2010:21:1083-8.

6. Gralla RJ, De Wit R, Herrstedt J, Carides AD, lanus J, Guoguang-Ma J, et al. Antiemetic efficacy of the neurokinin-1 antagonist, aprepitant, plus a 5-HT3 antagonist and a corticosteroid in patients receiving anthracy-clines or cyclophosphamide in addition to high-dose cisplatin: analysis of combined data from two phase III randomized clinical trials. Cancer. 2005;104:864-8.

7. Jordan K, Sippel C, Schmoll HJ. Guidelines for antiemetic treatment of chemotherapy-induced nausea and vomiting: past, present, and future recommendations. Oncologist. 2007;12:1143-50.

8. Liau CT, Chu NM, Liu HE, Deuson R, Lien J, Chen JS. Incidence of chemotherapy-induced nausea and vomiting in Taiwan: physicians' and nurses' estimation vs. patients' reported outcomes. Support Care Cancer. 2005;13:277-86

9. MASCC Antiemesis Tool@ (MAT) [http://www.mascc.org/mat]

10. Caracuel F, Muñoz N, Baños U, Ramirez G. Adherence to antiemetic guidelines and control of chemotherapy-induced nausea and vomiting (CINV) in a large hospital. J Oncol Pharm Pract. 2014. [Epub ahead of print].

11. Keat CH, Phua G, Abdul Kassim MS, Poh WK, Sriraman M. Can granisetron injection used as primary prophylaxis improve the control of nausea and vomiting with low-emetogenic chemotherapy? Asian Pac J Cancer Prev. 2013;14:469-73.

12. Jones JM, Qin R, Bardia A, Linquist B, Wolf S, Loprinzi CL. Antiemetics for chemotherapy-induced nausea and vomiting occurring despite prophylactic antiemetic therapy. J Palliat Med. 2011;14:810-4.

13. Bouganim N, Dranitsaris G, Hopkins S, Vandermeer L, Godbout L, Dent S, et al. Prospective validation of risk prediction indexes for acute and delayed chemotherapy-induced nausea and vomiting. Curr Oncol. 2012;19:e414-21.

14. Kris MG, Roila F, De Mulder PH, Marty M. Delayed emesis following anticancer chemotherapy. Support Care Cancer. 1998;6:228-32.

15. Kim HK, Hsieh R, Chan A, Yu S, Han B, Gao Y, et al. Impact of CINV in earlie cycles on CINV and chemotherapy regimen modification in subsequent cycles in Asia Pacific clinical practice. Support Care Cancer. 2014. [Epub ahead of print].

16. Molassiotis A, Stamataki Z, Kontopantelis E. Development and preliminary validation of a risk prediction model for chemotherapy-related nausea and vomiting. Support Care Cancer. 2013;21:2759-67.

\section{Submit your next manuscript to BioMed Central and take full advantage of:}

- Convenient online submission

- Thorough peer review

- No space constraints or color figure charges

- Immediate publication on acceptance

- Inclusion in PubMed, CAS, Scopus and Google Scholar

- Research which is freely available for redistribution 